\title{
Doğuştan çarpık ayak - Ponseti sonrası gelişmeler
}

\author{
Congenital clubfoot - advances after Ponseti
}

\author{
Murat Oto \\ Pamukkale Üniversitesi Tıp Fakültesi, Ortopedi ve Travmatoloji Anabilim Dalı, Denizli
}

PEV tedavisi, Ponseti metodunun bilinir hale gelmesiyle birlikte, son 15-20 yıldır radikal olarak değişmiştir. Ponseti; alçılama, breys kullanımı ve relapsın tedavisi için tibialis anterior tendon transferini 1963 yılında tanımlamış ${ }^{[1]}$ ve daha ayrıntılı açıklamalarını içeren yazısını 1972'de yayımlamıştır. ${ }^{2]}$ Tekniğin tüm önerileri bugün de geçerliliğini korumakta ve günümüzde bile, Ponseti ve meslektaşları tarafından iyi-mükemmel uzun dönem sonuçlar rapor edilmektedir. ${ }^{[3-6]} 2011$ yılında yapılan bir derlemede, Ponseti yönteminin mevcut yöntemler içinde en başarılı tedavi rejimi olduğu ve ilk düzeltme hızının \%90'lara ulaştığı rapor edilmiştir. ${ }^{[20]}$

Anahtar sözcükler: PEV; perkutan aşilotomi; tendon transferi; breys
Treatment of congenital clubfoot has changed radically with the introduction of Ponseti method in most of the pediatric orthopedic centers worldwide during the last 15 to 20 years. Ponseti first described his treatment regime including abduction bracing and tibialis anterior tendon transfer for the treatment of relapse in $1963,{ }^{[1]}$ and published a further detailed description in 1972.[2] All recommendations of Ponseti and his colleagues are still valid today and only minor adjustments have been made over the decades. The superior results of his method were reported by Ponseti and his colleagues in various long-term studies. ${ }^{[3-6]}$ According to a review performed in 2011, there is clear evidence that the Ponseti method is the most successful treatment regime available for congenital clubfoot, and reported an initial correction rate of around $90 \%$ in most treatments. ${ }^{[7]}$

Key words: CTEV; percutaneous achilotomy; tendon transfer; brace

\section{ETIYOLOJI VE RISKLER}

Hastalığın patofizyolojisine bakıldığında, genetik çalışmaların gün geçtikçe arttığını görmekteyiz. 2012 yılında Dobbs'un yaptığı genetik çalışmada, PEV'in poligenetik model içeren heterojen bir bozukluk olduğu belirtilmiştir. Geliş̧im yoluna bakıldığında, PITX1-TBX4 geçiş yolunun etkilendiğinin ve bu genlerin arka ayak tomurcuğu gelişimindeki öneminin altını çizmiştir. ${ }^{[8]}$

Risk faktörlerine yönelik 2015 yılında yayımlanan başka bir çalışmada, 'anne'ye ait sigara, kahve ve alkol kullanımının PEV gelişimine katkısı irdelenmiştir. Gebelik öncesi sigara içen ve gebelik esnasında da içmeye devam eden (özellikle ilk üç ayda) annelerin, bebeklerinde PEV riskini iki kat arttırdığı gösterilmiştir. Aşırı düzeyde olmadığı sürece, kahve ve alkol kullanımının risk oranına katkıda bulunmadığı rapor edilmiştir. ${ }^{[9]}$

Gelişimsel kalça displazisinin (GKD), PEV ile birlikte sık gözüktüğüne ait genel kanı ile ilgili, 2014 yılında yapılmış 21 yıllık ileriye dönük gözlemsel çalışmada ise, PEV ve GKD arasındaki korelasyon incelenmiş ve sonuç olarak GKD hastalarında PEV'in bir risk faktörü olarak görülmediği öne sürülmüştür. ${ }^{[10]}$

\section{MANIPÜLASYON VE ALÇI}

Ponseti, manipülasyon ve alçının nasıl yapılacağını kendi yazılarında çok ayrıntılı bir şekilde tanımlamışక̧,2] ve 1997 yılındaki yazısında, meslektaşları tarafından yapılan ortak hataları ortaya koyduğu makalesini yayımlamıştır. ${ }^{[11]}$ 2006'daki yazısında, alçının ince bir pamuk üzerine iyi bir şekilde mold edilerek yapılması gerektiğini tekrar hatırlatmış ve alçının kaymasının, mevcut deformitenin komplike hale gelmesindeki en önemli neden olduğunu vurgulamıştır. ${ }^{[12]} 2007$ yılında, yine Ponseti'nin

- Illetişim adresi: Doç. Dr. Murat Oto, Pamukkale Üniversitesi Tıp Fakültesi, Ortopedi ve Travmatoloji Anabilim Dalı, Denizli Tel: 0532 - 4271343 e-posta: muratoto@hotmail.com

- Geliş tarihi: 25 Mayıs 2015 Kabul tarihi: 25 Mayıs 2015 
kliniğinden Morcuende, alçı aşamasında sık yapılan bir hata olan, alçının bir gece öncesinden çıkarılması ile ilgili yazısında, yeni alçı yapılmadan hemen önce alçının çıkarılmasının gerekliliği üzerinde durmuş ve bir gece önceden çıkartılan alçılamalarda, düzelme için gereken sürenin ve alçı sayısının arttığını belirtmiştir. ${ }^{[13]}$

Ponseti protokolüne göre, alçı değişimi haftada bir alçılama şeklinde tanımlansa da, Morcuende, 2005 yılındaki çalışmasında, beş günde bir yapılan alçılama ile de benzer sonuçlar elde ettiğini bildirmiştir. ${ }^{[5]}$ Başka bir çalışmada ise, alçı değişiminin haftada üç kez yapıldığı ve haftada bir alçı değişimi yapılan grupla benzer sonuçlar elde edildiği yayımlanmıştır. ${ }^{[14]} 2015$ yılının başlarında yayımlanan başka bir çalışmada, 41 çocuğun 66 ayağında, haftada iki kez alçı yapılan 33 ayak ile ponseti protokolü uygulanan 33 ayak karşılaştırılmış ve hızlandırılmış tedavinin ilk düzeltme aşamasını hızlandırdığı ve en az klasik protokol kadar etkin olduğu bildirilmiştir. ${ }^{[15]}$ Pirani, bu konuda yaptığı manyetik rezonans (MR) görüntüleme çalışmasında, sık alçı değişiminin bu yaş grubundaki hızlı büyümeye adaptasyonu zorlaştıracağını bildirmiş ve yukarıdaki görüşe karşı fikir beyan etmiştir. ${ }^{[16]}$

Manipülasyon ve alçı tekniğine ait tamamiyle farklı bir öneri getiren, 2008 yilında JBJS Br'de (The Journal of Bone \& Joint Surgery [British Volume]) yayımlanmış bir çalışmada, 51 bebeğin 80 ayağında alçılama tekniği, diz altı softcast alçı kullanılarak modifiye edilmiştir. Ponseti'nin önerdiği şekilde manipülasyon yapılmış, tedavi doğumdan hemen sonra başlamış ve her alçılamada Pirani skoru not alınmıştır. Gerekli olduğu takdirde perkütan Aşil tenotomisi yapılmış ve sonrasında, çocuğun ayağı Denis Brown abduksiyon botlarına sığıncaya kadar alçıya devam edilmiştir. Bu yöntemde, Ponseti'den farklı olarak, ayak bileği ve talusun rotasyonunu engellemenin iyi oturtulmuş (molded) bir kısa bacak alçı ile de mümkün olacağını düşündüklerini ve kendi serilerinde sadece bir hastada alçı kayması problemi ile karşılaştıklarını bildirmişlerdir. Ponseti'nin uzun bacak alçılama önerisinin; yapılırken uzun sürmesinin, kasık bölgesinde hijyenik problemlere yol açma olasılığı bulunmasının ve çıkartırken alçı motoru ve bıçak gibi keskin aletlere ihtiyaç duyulmasının doğuracağı risklerin, bu yöntemle ortadan kalkacağını rapor etmişlerdir. Yazarlar, sonuçlarının ümit verici olduğunu belirtmişlerdir. ${ }^{[17]} \mathrm{Bu}$ çalışmaya karşıt görüş bildiren, 2013 yılına ait başka bir ileriye dönük randomize çalışmada, diz üstü ve diz altı alçılama yöntemleri karşılaştırılmış ve diz altı alçılamanın kabul edilemez ölçüde tedavi süresini uzattığı ve yüksek başarısızlık oranı olduğu bildirilmiştir. ${ }^{[18]}$

Alves, alçılamaya başlama yaşının önemini sorguladığı çalışmasında, hastalarını altı aydan önce ve altı aydan sonra tedaviye başlayanlar olarak iki gruba ayırmış ve yaygın kanının aksine yapılan alçı sayısında, başlangıç aşamasında oluşan düzeltme, rekürrens hızı ve tibialis anterior transferine ihtiyaç gibi hiçbir ana parametrede bir fark olmadığını bildirmiştir. ${ }^{[19]} 2010$ yılında yapılan bir çalışmada, başlamanın, ilk dört hafta sonrasına ve ayak büyüklüğünün en az $8 \mathrm{~cm}$ oluncaya kadar ertelenmesi gerektiği önerilmiştir. ${ }^{[20]}$ Ancak, bu konudaki genel kabul görmüş yaklaşımın, alçılamaya ilk bir ay içinde başlanması olduğuna dair literatür çoğunluktadır. ${ }^{21]}$ Esas olan, çocuğun, özellikle de prematür bebeklerin stabil hale gelmesi ve ailenin çocuğu düzenli olarak haftalık kontrollere getirebilmesidir. [21]

Tedavinin ilerlemesi ile ilgili monitorizasyonda ve perkütan aşilotominin zamanlamasının tayininde, ayağın ilk geldiği zaman ve takiplerinde, Pirani skorlanmasının kullanılmasının gözlemciler arası güvenilirliğinin çok yüksek olduğunu gösteren pek çok çalışma vardır. ${ }^{[22-24]}$ Her ne kadar bir çalışmada, düzeltme için ihtiyaç duyulan alçı sayısı ve başlangıçtaki Pirani skoru arasında düşük korelasyon gösterilse de, ${ }^{[25]}$ çalışmaların çoğunda, başlangıç Pirani skorları ile hastalığın şiddeti ve sonuçları arasında belirgin bir korelasyon mevcuttur. ${ }^{[26,27]}$ Tenotominin zamanlamasının tayininde radyografinin yerini sorgulayan 2014 yılına ait bir çalışmada, yazarlar, tenotomi öncesi çekilen ayak grafilerinin rekürrens ile ilişkisini araştırmışlar ve dorsifleksiyon grafisinin (ayak dorsifleksiyona zorlanmış şekilde çekilen yan grafi) rekürrens riskinin artışı ile doğru orantılı olduğunu tespit etmişlerdir. Tenotomi öncesi Ponseti yöntemi ile tedavi edilmiş ve $15^{\circ}$ 'nin üstünde dorsifleksiyona sahip olan ayaklarda rekürrens görülmezken, dorsifleksiyonu $15^{\circ}$ 'nin altındaki ayaklarda nüks gözlendiğini rapor etmişlerdir. ${ }^{[28]}$

\section{PERKÜTAN AŞiL TENOTOMISI (PAT)}

PAT, Ponseti metodunun ana komponentlerinden biri olup, Ponseti'nin ilk çalışmasında da tedavinin tamamlanmasında PAT'ın \%79 gibi büyük bir oranda önemi olduğu vurgulanmıştır. ${ }^{[1]}$ Ponseti, PAT’ı lokal anestezi altında yapmayı önermiştir. ${ }^{[3]}$ Benzer şekilde başka bir çalışmada, PAT'ın poliklinik şartlarında yapılmasının etkili ve güvenli olduğu bildirilmiştir. ${ }^{[29]} \mathrm{Bu}$ hasta grubundaki tenotomiler, ortalama 9,5 haftalık çocuklara yapılmış ve yazarlar, poliklinik şartlarında yapılan tenotomilerin tam yapılmasının önemini vurgulamışlardır. Bor ve arkadaşları, 2007 yılında yaptıkları çalışmalarında, genel anestezi yerine hafif sedasyon verilmesini önermişler, lokal anestezinin, yenidoğandan süt çocuğuna geçiş döneminde çocuğu sakinleştirmekte yetersiz olduğunu rapor etmişlerdir. ${ }^{\left[{ }^{30]}\right.}$ Başka bir çalışmada, havayolu için bir enstrüman kullanılmaksızın, propofol ile sedasyon yapmanın yeterli olacağını vurgulamışlardır. 
[31] 2009 yılında literatüre giren başka bir çalışmada, 89 hastanın 182 ayağına genel anestezi altında tenotomi yapılmış ve anesteziye ait hiçbir komplikasyon gelişmediği bildirilmiştir. ${ }^{[32]}$ Aynı çalışmanın ayrıntılarına bakıldığında, Parada ve arkadaşlarının, genel anestezi verdikleri hastalar için ameliyat sonrası en az dört saat monitorizasyon ve yakın takip önerdiklerini görmekteyiz. ${ }^{[32]} 2014$ yılında, 32 bebeğin 56 ayağının incelendiği literatüre tamamiyle yeni bir yaklaşım öneren başka bir çalışmada, hastaları manipülasyon ve dizüstü breys kullanılarak tedavi yoluna gidilmiş, alçı ve tenotomi basamakları ekarte edilmiş ve ortalama dört ayda ayakların düzeldiği ve hiçbir hastanın aşilotomiye ihtiyaç duymadığı vurgulanmıştır. ${ }^{[33]}$

Ponseti metodundaki kritik basamaklardan birisi de, tenotominin zamanlamasıdır. Ponseti, ayak en az $60^{\circ}$ abduksiyona ulaştığında ve dorsifleksiyonun $15-20^{\circ}$ 'nin altında olduğu durumlarda tenotomi önermiştir. Abduksiyonun $60^{\circ}$ 'nin altında olması, subtalar dizilimin sağlanmasını engeller ve topuğun varusu ayağın supinasyonu düzeltilmeden yapılan aşilotomi, 'rocker bottom' ayak deformitesini oluşturur. 2007 yılında Radler ve arkadaşlarının yaptığı radyolojik çalışmada, subtalar derotasyonun göstergeleri olan talokalkaneal açının (ön-arka/yan grafilerde) tenotomiden etkilenmediği ve bu nedenle, tenotomi yapılmadan önce bu açıların düzeltilmesinin esas olduğu vurgulanmıştır. ${ }^{[34]}$ Tenotomi öncesi ayağın yeterli miktarda düzelip düzelmediğini ölçmenin yollarından biri olan Pirani skorlamasını sorgulayan başka bir çalışmada; MFS (orta ayak skoru)'nin 1'in altında, HFS (arka ayak skoru)'nun 1 'in üstünde olduğu durumların tenotomi endikasyonu oluşturacağı bildirilmiştir. ${ }^{[23]}$

Tenotominin tekniğine geldiğimizde; 2015 yılında basılmış bir çalışmada, Aşil tendonunun kesilmesi esnasında tendonun tamamının kesilememesinin veya 'pop' sesinin duyulamamasının, eğer hastada $10-20^{\circ}$ arası dorsifleksiyona ulaşıldıysa nihai sonuca etki etmediği gösterilmiştir. ${ }^{[35]}$

Tekniğe ait ayrıntıların, daha çok tenotomi komplikasyonlarını ortaya koyan çalışmalarda tanımlandığını görüyoruz. ${ }^{[36,37]}$ Dobbs, çalışmasında, 200 ayağın dördünde peroneal damarlara bağlı olduğunu düşündüğü ciddi kanama yaşadığını ifade etmiş;; ${ }^{[36]}$ Burghardt ise, sekiz haftalık bir bebekte tenotomi sonrası psödoanevrizma geliştiğini rapor etmiştir ${ }^{[37]}$ Nörovasküler yapıların açık eksplorasyonu, arterin bağlanması ve primer sinir tamiri gerektirecek kadar majör yaralanmaların olduğunu ifade eden başka bir çalışma da mevcuttur. ${ }^{[38]}$

Pek çok çalışmada, tenotomi sonrası iyileşme fazla$r ı$ incelenmiş ve üç hafta içinde tendon devamlılı̆̆ının sağlandığı gösterilmiştir. ${ }^{[39-41]}$ Bacak kaslarındaki atrofinin primer nedeninin fetal dönem olduğu, tedavinin basamakları olan alçı veya tenotominin öncesinde de bunun var olduğuna başka bir çalışmada değinilmiştir. ${ }^{[42]}$

\section{BREYSLEME}

Ponseti, ilk yazısında, ayak abduksiyon ortezini üç ay hiç çıkarmadan taktığını ve sonrasında ortalama 21 ay daha kullandığını ve bu olgularda \%56 rekürrens bulduğunu bildirmiştir. ${ }^{[1]}$ Bu yüksek oran nedeniyle, ikinci yazısında bu süreyi 5-6 yaşa kadar uzattığını ve daha az rekürrens gördügüunü belirtmiştir. ${ }^{[2]}$ illk düzeltme aşamasından (alçılama ve tenotomi) sonra, düzeltmenin korunması aşamasının kritik faktörü olan breysleme aşamasında en büyük risk faktörü, ailenin eğitim düzeyi ve tedaviye uyum yeteneğidir. ${ }^{[43]}$ Uzun dönem sonucu belirleyen en önemli faktörlerden birinin abduksiyon ortezi kullanımı olduğu gerçeği, ortez çeşitleri ve kullanım süresi ile ilgili pek çok çalışma yapılmasına neden olmuştur. Çocuğun tedaviye uyumunu sağlamak için farklı cihazlar geliştirilmiştir. Ayakkabılar arasında, esnek bara sahip ilk esnek (flexible) breys, Kessler tarafından tanıtılmıştır. ${ }^{[44]}$ Garg ve Porter, Dobbs tarafından tasarlanan dinamik ortezle ilgili sonuçlarını 2009'da yayımlamışlardır. Bu dinamik breysle; artmış uyum, daha az rekürrens, azalmış cilt komplikasyonları ve klasik breyslere göre azalmış cerrahi gereksinimi rapor etmişlerdir. ${ }^{[45]} 2010$ yılında yapılan başka bir çalışma, breys tedavisine uyumu arttırmaya odaklanmış ve aile eğitimi, aile ile cesaretlendirici görüşmeler, yazılı öneriler vermenin, aile ile güçlü bağ kurmanın breysleme aşamasında uyumu arttırdığını rapor etmiştir. ${ }^{[46]}$

Geleneksel abduksiyon breysleme yönteminde, barın ucuna bağlanmış iki ayakkabı vardır; ancak, hem bazı hekimler hem de aileler, tek taraflı olgularda unilateral breys kullanmanın daha konforlu olacağına dair bir inanış içindedirler. Bu sorunun cevabını sorgulayan çalışmalarda, tek taraflı breys kullanmanın rekürrens riskini arttırdığg[ ${ }^{[4]}$ ve AFO (ayak - ayak bileği ortezi) kullanmanın da bu riski arttıran bir başka yöntem olduğu gösterilmiştir. ${ }^{[48]}$ Farklı tipte abduksiyon ortezleri tanımlanmış ve bu farklı, yeni ve pahalı breyslerin kullanım kolaylığı sağladığı, ancak klinik olarak daha iyi sonuçlar ortaya koymadığı, çalışmalarla gösterilmiştir. ${ }^{[49,50]}$

Breysleme aşamasında kullanılan klasik, 'dinamikolmayan' breyslerin kullanımına ait uzun dönem sonuçlar literatürde yerini almışken (1-6), aynı durum farklı biyomekanik özelliklere sahip breysler için söz konusu değildir. 
Morcuende, 2012 yılında, hastaya özel breyslemeyi sorguladığı, beş yaşında bir çocuğun eklem yapısını ve kinematiğini taklit edebilen maket modelinde yapılan ve biyomekanik temellere dayanan çalışmasında, breys kullanıldığında ve istirahatte kas-tendon gerginliğini karşılaştırmıştır. Bu çalışmanın sonucunda, bu biyomodellemenin etkin olduğunu ve gelecekte hastaya özel breyslerin uyumu arttıracağını düşündüğünü ifade etmiştir. ${ }^{[51]}$

Morcuende ve arkadaşları, 2011 yılında yaptıkları çalışmalarında; sözü geçen bütün teknik özelliklerin önemi bir yana, her zaman en önemli parametrenin, breyslemeye uyum aşamasında ailenin bu aşamanın ne kadar önemli olduğu ve doğru kullanımı konusundaki eğitimi olduğunu ifade etmişlerdir. ${ }^{[52]}$ Breys kullanımının rotasyonel problemlere yol açma potansiyelinin sorgulandığı başka bir çalışmada, breysin femur ve tibiada torsiyonel bir problem yaratmadığı gösterilmiştir. ${ }^{[53]}$

\section{PONSETI METODUNUN SINIRLARI}

Ponseti'nin 1963'teki ilk makalesinde, en büyük hastanın altı aylık olduğu ve bu çalışmaya artrogripotik ve/veya sendromik bebeklerin dahil edilmediği görülmektedir. O zamandan bu yana pek çok çalışma, daha büyük çocukların tedavisini, cerrahi sonrası bozulan ayakların tedavisini ve sendromlu çocukların PEV'ini incelemiştir.

Bor ve arkadaşları, alçı sonrası bozulmuş veya başvuru zamanı üç aydan büyük 36 ayağa ait çalışmalarında, açık cerrahi gereksinimi olmadan \%97 başarı bildirmişlerdir. ${ }^{[54]}$ Lourenço ve Morcuende, ihmal edilmiş 24 ayağı içeren çalışmalarında (ortalama yaş, 3,9 ), olguların $\% 67$ 'sinde iyi düzelme rapor etmişlerdir. ${ }^{[55]}$ Benzer şekilde cesaretlendirici sonuçlar, Spiegel ve arkadaşları tarafından 260 ayağın incelendiği çalışmada da bildirilmiştir. Bu çalışmada, 1-6 yaş arası başvuran hastaların Ponseti metodu ile tedavisinin sonuçları irdelenmiştir. Hastaların \%79'unda, tam düzelme için PAT'ın yeterli, \%14'ünde ise açık cerrahi ihtiyacı olduğunu; sonuç olarak da, ilk düzeltmenin (plantigrad ayak) altı yaşına kadar olan çocuklarda büyük oranda yeterli ve başarılı olduğunu rapor etmişlerdir. ${ }^{[56]}$

Ek olarak, daha ileri yaş veya ihmal edilmiş PEV hastalarında ve posteromediyal gevşetme yapılmış hastalarda, Ponseti protokolü uygulanarak, \%86 hastada $(71 / 83)$ plantigrad ve tam düzelmiş ayak elde edilmiştir. ${ }^{[57]}$ Morcuende'nin kendi kliniğindeki artrogripotik ayaklarda yapılan Ponseti tedavisi sonucunda, \%16 hastada ilk düzelme sonrası açık cerrahiye giden relaps gözlenmiştir. ${ }^{[58]}$
Miyelomeningosel tanısı olan PEV'lerle yapılan bir çalışmada, yedi ay sonrası relaps oranı \%68 olarak gösterilmiş̧, ${ }^{[59]} 2012$ yılında yapılmış ileriye dönük başka bir çalışmada, idiyopatik olmayan ayakların Ponseti ile tedavisi sonrası \%44 nüks görülmüş, ancak yazının sonuç bölümüne bakıldığında, bu orana rağmen tüm idiyopatik olmayan ayaklarda Ponseti yönteminin kullanılması önerilmiştir. ${ }^{[60]}$

\section{SONUÇ}

Her ne kadar, etiyolojiden bağımsız olarak, tedavi metodları hastaların pek çoğunda etkinlik gösterse de, etiyoloji hakkında bilgilerimizin artması, prognozu, eşlik eden anomalilere ait riskleri ve tedaviye cevabı etkileyecektir. Patofizyoloji-genetik ile relaps arasında korelasyon kurulabilirse, kişiye özel-etiyolojiye yönelik tedavi, breys giyme süresini azaltacaktır. Tedavinin esas amacının, tam fonksiyonel ve ağrısız bir ayak olduğunu unutmamak ve buna ulaşmak için gerekirse farklı yaklaşımların (Ponseti ve Fransız metodu) kombinasyonunu kullanmaya yetecek düzeyde bilgili ve tecrübeli olmak gerekmektedir.

\section{KAYNAKLAR}

1. Ponseti IV, Smoley EN. The classic: Congenital club foot: the results of treatment. Clin Orthop Relat Res 2009;467(5):1133-45. CrossRef

2. Ponseti IV, Campos J. Observations on pathogenesis and treatment of congenital club foot. Clin Orthop Relat Res 1972;84:50-60.

3. Laaveg SJ, Ponseti IV. Long-term results of treatment of congenital club foot. J Bone Joint Surg Am 1980;62(1):23-31.

4. Cooper DM, Dietz FR. Treatment of idiopathic club foot. A thirty-year follow-up note. J Bone Joint Surg Am 1995;77(10):1477-89.

5. Morcuende JA, Abbasi D, Dolan LA, Ponseti IV. Results of an accelerated Ponseti protocol for clubfoot. J Pediatr Orthop 2005;25(5):623-6.

6. Morcuende JA, Dolan LA, Dietz FR, Ponseti IV. Radical reduction in the rate of extensive corrective surgery for club foot using the Ponseti method. Pediatrics 2004;113(2):376-80.

7. Jowett CR, Morcuende JA, Ramachandran M. Management of congenital talipes equinovarus using the Ponseti method: a systematic review. J Bone Joint Surg Br 2011;93(9):1160-4. CrossRef

8. Dobbs MB, Gurnett CA. Genetics of clubfoot. J Pediatr Orthop B 2012;21(1):7-9. CrossRef

9. Werler MM, Yazdy MM, Kasser JR, Mahan ST, Meyer RE, Anderka M, Druschel CM, Mitchell AA. Maternal cigarette, alcohol, and coffee consumption in relation to risk of clubfoot. Paediatr Perinat Epidemiol 2015;29(1):3-10. doi: 10.1111/ppe.12163

10. Paton RW, Choudry QA, Jugdey R, Hughes S. Is congenital talipes equinovarus a risk factor for pathological dysplasia of the hip?: a 21-year prospective, longitudinal observational study. Bone Joint J 2014;96-B(11):1553-5. CrossRef 
11. Ponseti IV. Common errors in the treatment of congenital clubfoot. Int Orthop 1997;21(2):137-41.

12. Ponseti IV, ZhivkovM, Davis N, SinclairM, Dobbs MB, Morcuende JA. Treatment of the complex idiopathic clubfoot. Clin Orthop Relat Res 2006;451:171-6.

13. Terrazas-Lafargue G, Morcuende JA. Effect of cast removal timing in the correction of idiopathic clubfoot by the Ponseti method. lowa Orthop J 2007;27:24-7.

14. Harnett P, Freeman R, HarrisonWJ, Brown LC, Beckles V. An accelerated Ponseti versus the standard Ponseti method: a prospective randomised controlled trial. J Bone Joint Surg Br 2011;93(3):404-8. CrossRef

15. Elgohary HS, Abulsaad M. Traditional and accelerated Ponseti technique: a comparative study. Eur J Orthop Surg Traumatol 2015;25(5):949-53. CrossRef

16. Pirani S, Zeznik L, Hodges D. Magnetic resonance imaging study of the congenital clubfoot treated with the Ponseti method. J Pediatr Orthop 2001;21(6):719-26.

17. Brewster MB, Gupta M, Pattison GT, Dunn-van der Ploeg ID. Ponseti Casting: a new soft option. J Bone Joint Surg Br 2008;90(11):1512-5. CrossRef

18. Maripuri SN, Gallacher PD, Bridgens J, Kuiper JH, Kiely NT. Ponseti casting for club foot -above- or below-knee?: A prospective randomised clinical trial. Bone Joint J 2013;95B(11):1570-4. CrossRef

19. Alves C, Escalda C, Fernandes P, Tavares D, Neves MC. Ponseti method: does age at the beginning of treatment make a difference? Clin Orthop Relat Res 2009;467(5):1271-7. CrossRef

20. Iltar $\mathrm{S}$, Uysal $\mathrm{M}$, Alemdaroğlu $\mathrm{KB}$, Aydoğan $\mathrm{NH}$, Kara $\mathrm{T}$, Atlihan D. Treatment of clubfoot with the Ponseti method: should we begin casting in the newborn period or later? J Foot Ankle Surg 2010;49(5):426-31. CrossRef

21. Böhm S, Sinclair M. Report of the 1 st European consensus meeting on Ponseti clubfoot treatment. J Child Orthop 2013;7(3):251-4. CrossRef

22. Flynn JM, Donohoe M, Mackenzie WG. An independent assessment of two clubfoot-classification systems. J Pediatr Orthop 1998;18(3):323-7.

23. Shaheen $\mathrm{S}$, JaiballaH, Pirani $\mathrm{S}$. Interobserver reliability in Pirani clubfoot severity scoring between a paediatric orthopaedic surgeon and a physiotherapy assistant. J Pediatr Orthop B 2012;21(4):366-8. CrossRef

24. Dyer PJ, Davis N. The role of the Pirani scoring system in the management of club foot by the Ponseti method. J Bone Joint Surg Br 2006;88(8):1082-4.

25. Chu A, Labar AS, Sala DA, van Bosse HJ, Lehman WB. Club foot classification: correlation with Ponseti cast treatment. J Pediatr Orthop 2010;30(7):695-9. CrossRef

26. Zhang W, Richards BS, Faulks ST, Karol LA, Rathjen KA, Browne $\mathrm{RH}$. Initial severity rating of idiopathic clubfeet is an outcome predictor at age two years. J Pediatr Orthop B 2012;21(1):16-9. CrossRef

27. Goriainov V, Judd J, Uglow M. Does the Pirani score predict relapse in clubfoot? J Child Orthop 2010;4(5):439-44. CrossRef

28. O'Halloran CP, Halanski MA, Nemeth BA, Zimmermann CC, Noonan KJ. Can Radiographs Predict Outcome in Patients With Idiopathic Clubfeet Treated With the Ponseti Method? J Pediatr Orthop 2014. [Epub ahead of print]

29. Lebel E, Karasik M, Bernstein-Weyel M, Mishukov Y, Peyser A. Achilles tenotomy as an office procedure: safety and efficacy as part of the Ponseti serial casting protocol for clubfoot. J Pediatr Orthop 2012;32(4):412-5. CrossRef
30. Bor N, Katz Y, Vofsi O, Herzenberg JE, Zuckerberg AL. Sedation protocols for Ponseti clubfoot Achilles tenotomy. J Child Orthop 2007;1(6):333-5. CrossRef

31. Iravani M, Chalabi J, Kim R, Ebramzadeh E, Zionts LE. Propofol sedation for infants with idiopathic clubfoot undergoing percutaneous tendoachilles tenotomy. J Pediatr Orthop 2013;33(1):59-62. CrossRef

32. Parada SA, Baird GO, Auffant RA, Tompkins BJ, Caskey PM. Safety of percutaneous tendoachilles tenotomy performed under general anesthesia on infants with idiopathic clubfoot. J Pediatr Orthop 2009;29(8):916-9. CrossRef

33. Su Y, Nan G. Manipulation and brace fixing for the treatment of congenital clubfoot in newborns and infants. BMC Musculoskelet Disord 2014;15:363. CrossRef

34. Radler C, Manner HM, Suda R, Burghardt R, Herzenberg JE, Ganger R, Grill F. Radiographic evaluation of idiopathic clubfeet undergoing Ponseti treatment. J Bone Joint Surg Am 2007;89(6):1177-83.

35. Karami M, Dehghan P, Moshiri F, Shamami MS. Effect of unintentional partial Achilles tenotomy on Ponseti clubfoot management outcomes. J Pediatr Orthop B 2015;24(1):1-5. CrossRef

36. Dobbs MB, Gordon JE, Walton T, Schoenecker PL. Bleeding complications following percutaneous tendoachilles tenotomy in the treatment of clubfoot deformity. J Pediatr Orthop 2004;24(4):353-7.

37. Burghardt RD, Herzenberg JE, Ranade A. Pseudoaneurysm after Ponseti percutaneous Achilles tenotomy: a case report. J Pediatr Orthop 2008;28(3):366-9. CrossRef

38. Changulani M, Garg NK, Rajagopal TS, Bass A, Nayagam SN, Sampath J, Bruce CE. Treatment of idiopathic club foot using the Ponseti method. Initial experience. J Bone Joint Surg Br 2006;88(10):1385-7.

39. BarkerSL, Lavy CB. Correlation of clinical and ultrasonographic findings after Achilles tenotomy in idiopathic club foot. J Bone Joint Surg Br 2006;88(3):377-9.

40. Maranho DA, Nogueira-Barbosa MH, SimãoMN, Volpon JB. Ultrasonographic evaluation of Achilles tendon repair after percutaneous sectioning for the correction of congenital clubfoot residual equinus. J Pediatr Orthop 2009;29(7):80410. CrossRef

41. Mangat KS, Kanwar R, Johnson K, Korah G, Prem H. Ultrasonographic phases in gap healing following Ponseti-type Achilles tenotomy. J Bone Joint Surg Am 2010;92(6):1462-7. CrossRef

42. Ippolito E, De Maio F, Mancini F, Bellini D, Orefice A. Leg muscle atrophy in idiopathic congenital clubfoot: is it primitive or acquired? J Child Orthop 2009;3(3):171-8. CrossRef

43. Dobbs MB, Rudzki JR, Purcell DB, Walton T, Porter KR, Gurnett CA. Factors predictive of outcome after use of the Ponseti method for the treatment of idiopathic clubfeet. J Bone Joint Surg Am 2004;86-A(1):22-7.

44. Kessler JI. A new flexible brace used in the Ponseti treatment of talipes equinovarus. J Pediatr Orthop B 2008;17(5):24750. CrossRef

45. Garg S, Porter K. Improved bracing compliance in children with clubfeet using a dynamic orthosis. J Child Orthop 2009;3(4):271-6. CrossRef

46. Zionts LE, Dietz FR. Bracing following correction of idiopathic clubfoot using the Ponseti method. J Am Acad Orthop Surg 2010;18(8):486-93 
47. George HL, Unnikrishnan PN, Garg NK, Sampath J, Bruce CE. Unilateral foot abduction orthosis: is it a substitute for Denis Browne boots following Ponseti technique? J Pediatr Orthop B 2011;20(1):22-25. CrossRef

48. Janicki JA, Wright JG, Weir S, Narayanan UG. A comparison of ankle foot orthoses with foot abduction orthoses to prevent recurrence following correction of idiopathic clubfoot by the Ponseti method. J Bone Joint Surg Br 2011;93(5):700-4. CrossRef

49. Hemo Y, Segev E, Yavor A, Ovadia D Wientroub S, Hayek S. The influence of brace type on the success rate of the Ponseti treatment protocol for idiopathic clubfoot. J Child Orthop 2011;5(2):115-9. CrossRef

50. Zionts LE, Frost N, Kim R, Ebramzadeh E, Sangiorgio SN. Treatment of idiopathic clubfoot: experience with the Mitchell-Ponseti brace. J Pediatr Orthop 2012;32(7):706-13. CrossRef

51. Dimeo AJ Sr, Lalush DS, Grant E, Morcuende JA. Development of a surrogate biomodel for the investigation of clubfoot bracing. J Pediatr Orthop 2012;32(7):e47-52. CrossRef

52. Paulsen-Miller M, Dolan LA, Stineman A, Morcuende JA. Understanding the educational needs for parents of children with clubfoot. Orthop Nurs 2011;30(4):273-8; quiz 279-80. CrossRef

53. Boehm S, Sinclair M. Foot abduction brace in the Ponseti method for idiopathic clubfoot deformity: torsional deformities and compliance. J Pediatr Orthop 2007;27(6):712-6.
54. Bor N, Herzenberg JE, Frick SL. Ponseti management of clubfoot in older infants. Clin Orthop Relat Res 2006;444:224-8.

55. Lourenço AF, Morcuende JA. Correction of neglected idiopathic club foot by the Ponseti method. J Bone Joint Surg Br 2007;89(3):378-81.

56. Spiegel DA, Shrestha OP, Sitoula P, Rajbhandary T, Bijukachhe B, Banskota AK. Ponseti method for untreated idiopathic clubfeet in Nepalese patients from 1 to 6 years of age. Clin Orthop Relat Res 2009;467(5):1164-70. CrossRef

57. Nogueira MP, Ey Batlle AM, Alves CG. Is it possible to treat recurrent clubfoot with the Ponseti technique after posteromedial release?: a preliminary study. Clin Orthop Relat Res 2009;467(5):1298-305. CrossRef

58. Morcuende JA, Dobbs MB, Frick SL. Results of the Ponseti method in patients with clubfoot associated with arthrogryposis. lowa Orthop J 2008;28:22-6.

59. Gerlach DJ, Gurnett CA, Limpaphayom N, Alaee F, Zhang Z, Porter K, Kirchhofer M, Smyth MD, Dobbs MB. Early results of the Ponseti method for the treatment of clubfoot associated with myelomeningocele. J Bone Joint Surg Am 2009;91(6):1350-9. CrossRef

60. Moroney PJ, Noël J, Fogarty EE, Kelly PM. A singlecenter prospective evaluation of the Ponseti method in nonidiopathic congenital talipes equinovarus. J Pediatr Orthop 2012;32(6):636-40. CrossRef 